\title{
Lockean Beliefs, Dutch Books, and Scoring Systems
}

\author{
Daniel Rothschild ${ }^{1}$
}

Received: 8 December 2020 / Accepted: 31 May 2021

(c) The Author(s) 2021

\begin{abstract}
On the Lockean thesis one ought to believe a proposition if and only if one assigns it a credence at or above a threshold (Foley in Am Philos Q 29(2):111-124, 1992). The Lockean thesis, thus, provides a way of characterizing sets of all-or-nothing beliefs. Here we give two independent characterizations of the sets of beliefs satisfying the Lockean thesis. One is in terms of betting dispositions associated with full beliefs and one is in terms of an accuracy scoring system for full beliefs. These characterizations are parallel to, but not merely derivative from, the more familiar Dutch Book (de Finetti in Theory of probability, vol 1, Wiley, London, 1974) and accuracy (Joyce in Philos Sci 65(4):575-603, 1998) arguments for probabilism.
\end{abstract}

\section{Introduction}

The Lockean thesis is a way of connecting all-or-nothing beliefs to graded beliefs (Foley 1992). On the Lockean thesis to have an all-or-nothing belief in a proposition is just to assign that proposition a credence at or above a certain threshold. ${ }^{1}$ One of the interesting and controversial features of Lockeanism is that it does not require that one's beliefs either be closed under logical implication or even be logically consistent. For a failure of closure note that at any threshold less than one it is possible, on the Lockean thesis, to believe two atomic propositions $P$ and $Q$ while not believing their conjunction $P \& Q{ }^{2}$ For a failure of consistency note that at a threshold of .6 it is possible to believe two propositions $P$ and $Q$, while also believing the negation of their conjunction, $\neg(P \& Q) .^{3}$

\footnotetext{
1 Sometimes the Lockean thesis is framed as one about what one ought to believe, rather than about what one does believe. The difference is not essential here.

2 See Leitgeb (2014) for an exploration of constraints, other than having a threshold of 1, on credences and thresholds that ensure closure (and consistency).

${ }^{3}$ Assume you assign a credence of .6 to $P$ and .6 to $Q$, then you can assign at most .8 to $\neg(P \& Q)$.
}

Daniel Rothschild

d.rothschild@ucl.ac.uk

1 University College London, London, UK 
We might want to ask what constraints the Lockean thesis does put on one's beliefs. Some constraints are well-known: for example, if the threshold for belief is greater than .5 , then, on the Lockean thesis, one cannot hold pairwise inconsistent beliefs. ${ }^{4}$ However, these constraints do not give necessary and sufficient conditions for satisfying the Lockean thesis. For, as we will see in Sect. 3, there are sets of beliefs that are pairwise consistent but that cannot be believed by a Lockean with a threshold greater than one-half. Pairwise consistency is thus a necessary but not sufficient condition for the Lockean thesis with a threshold greater than one-half.

In this paper, I present two types of characterizations of sets of beliefs for Lockean agents. I draw on a pair of related traditions for characterizing graded beliefs. The first tradition is that of the Dutch Book argument for probabilism. In this tradition, graded beliefs can be characterized by how they rationalize bets. Very roughly speaking, the Dutch Book argument establishes that a set of numerically graded beliefs is probabilistically coherent if and only if there is no collection of bets it rationalizes that leads to a sure loss. ${ }^{5}$ The second tradition is that of the accuracy argument for probabilism. In this tradition, numerical credences are given scores corresponding to their accuracy. ${ }^{6}$ The standard genre of result in this area is to establish that the numerical credences that are probabilistically coherent are equivalent to those that are not dominated in all worlds by another set of credences.

In the case of graded beliefs, both Dutch Book arguments and accuracy arguments are used to characterize probabilistically coherent beliefs. What I show here is that these arguments can also be used to characterize all-or-nothing beliefs satisfying the Lockean hypothesis.

It is worth noting that such a characterization is not directly extractable from the current literature. For accuracy arguments and Dutch Book arguments characterize probabilistically coherent numerical credences, and probabilistically coherent beliefs have a many-to-one relationship to Lockean belief sets.

What I give here is a direct characterization of Lockeanism via betting dispositions and accuracy scoring rules. To do this I propose mappings from sets of all-ornothing beliefs to betting dispositions and scores and, I show that, relative to these mappings, Lockean belief sets can be characterized by properties of betting dispositions and scores.

In the accuracy tradition there is already a small literature that addresses how the Lockean thesis fares vis-a-vis accuracy arguments. ${ }^{7}$ This paper advances that literature by providing more general results than previously available and by proving, as a corollary of these results, the main conjecture of Easwaran (2016). There is, by contrast, no literature that I am aware of relating Dutch Book arguments to the Lockean

\footnotetext{
4 See, e.g., Hawthorne and Bovens (1999) for this and related observations.

5 This argument goes back to Ramsey (1926) and de Finetti (1974). See Pettigrew (2019) for a recent book-length review and discussion.

6 See, e.g., Joyce (1998) and Pettigrew (2016a). This work is, in turn, dependent on the tradition of scoring graded predictions for their accuracy going back at least to Brier (1950); see Gneiting and Raftery (2007) for a recent discussion.

7 For example, Easwaran and Fitelson (2015), Pettigrew (2016b), Easwaran (2016) and Dorst (2019).
} 
account of belief. This paper fills that lacuna by giving two Dutch Book results for the Lockean account of belief.

The significance of these results varies depending on your starting assumptions. For example, these results show that if a set of full beliefs satisfy certain decisiontheoretic properties relative to a range of scoring systems then they are compatible with the Lockean thesis. If you think of full belief as basic and use Lockeanism as a way to derive ranges of graded beliefs from them (as in Easwaran 2016), then these results gives you the conditions on which this is possible. Likewise, if, as in classical works in decision theory, you take certain kinds of betting dispositions as basic, these results give necessary conditions for the dispositions to be associated with sets of full beliefs. More generally, these results make connections between three ways of thinking about epistemic commitments: graded beliefs, full beliefs, and betting dispositions.

Here is the plan: In the next Sect. 2, I outline the basic formal framework for modeling full beliefs. In Sect. 3, I define three different ways in which sets of full beliefs can be said to satisfy the Lockean thesis. In Sect. 4, I discuss ways of deriving betting dispositions from full beliefs and what it takes for those dispositions to be subject to a Dutch Book. In Sect. 5, I give an accuracy scoring system for full beliefs and state some standard decision-theoretic properties of full belief sets relative to the system. In the main section, Sect. 6, I give results linking these different ways of thinking about full beliefs. I discuss the significance of these results in Sect. 7 and outline a few directions for future work in Sect. 8.

\section{Framework}

Let $W$ be a finite set of worlds, which we will enumerate $w_{1} \ldots w_{m}$, let $P$ denote the set of proposition $2^{W},{ }^{8}$ enumerated $p_{1} \ldots p_{n}$, with $n=2^{m}$. Let $B \subseteq P$ be a subset of $o$ beliefs, $o \leq n$, enumerated $b_{1} \ldots b_{o}$. Let $t$ be a real number, $0<t \leq 1$, which we'll call the threshold. We think of $B$ as representing the set of propositions an agent believes (and hence $P \backslash B$ is the set of propositions the agent doesn't believe). In the next three sections we define various properties that $B$ can have, always in terms of the threshold $t$. These properties fall into three categories: Lockean properties, betting properties, and accuracy properties.

\section{Lockean Belief Sets}

On the Lockean thesis one should believe a proposition $p$ if and only if you assign it a credence greater than or equal to some threshold $t$. In this section we will define three different versions of the Lockean thesis.

\footnotetext{
${ }^{8}$ Notation: $2^{X}$ is the powerset of $X$.
} 
Let us begin by defining credences. A CREDENCE FUNCTION is a function $c$ from $P$ to $[0,1]$ such that $c(W)=1, c(\emptyset)=0$ and for any $X \subseteq W, c(X)=\sum_{w \in W} c(\{w\}){ }^{9}$

Since we use linear algebra to prove most of our main results it will be useful to rephrase some of the notions in terms of vectors. We correspond to each proposition $p$ an $m$-dimensional vector $\mathbf{p}=\left\langle p_{1}, \ldots, p_{m}\right\rangle$ such that

$$
p_{i}=\left\{\begin{array}{ll}
1 & \text { if } w_{i} \in p \\
0 & \text { otherwise }
\end{array} .\right.
$$

We correspond to the credence function $c$ the vector $\mathbf{c}=\left\langle c_{1} \ldots c_{m}\right\rangle$ such that $c_{i}=c\left(\left\{w_{i}\right\}\right)$. In this case it is easy to see that $c(p)=\mathbf{c} \cdot \mathbf{p} .{ }^{10}$

We begin with the weakest sense in which $B$ can satisfy the Lockean thesis relative to the threshold $t$. The set of beliefs $B$ is Lockean Compatible relative to the threshold $t$ iff there is a credence function $c$ such that: $c(b) \geq t$ (or $\mathbf{c} \cdot \mathbf{b} \geq t$ ) for all $b \in B$.

To say that $B$ is Lockean Compatible is not equivalent to saying that $B$ can be a Lockean's total set of beliefs. For if a Lockean's belief set $B$ contains two propositions $x$ and $y$ it also ought to contain $x \cup y$, but Lockean Compatibility does not guarantee this.

For this reason, we will define two stronger notions as well. The set of beliefs $B$ is LOCKEAN COMPLETE at threshold $t$ iff there is a credence function $c$ such that $c(p) \geq t$ iff $p \in B$ for all $p \in P$. We will also use a weakening of Lockean Completeness to allow cases where belief is optional at the threshold $t$. We say $B$ is Almost LockeAN COMPLETE relative to a threshold $t$ iff there is a credence function $c$ such that for all $p \in P$ if $c(p)>t$ then $p \in B$ and if $c(p)<t$ then $p \notin B$. As we shall see, this last notion connects more naturally with the decision-theoretic notions we define in relation to accuracy scoring.

We can now prove the claim in the introduction that there are sets of beliefs that are pairwise compatible but are not holdable by the Lockean with $t>.5$. A belief set $B$ is PAIRWISE COMPATIBLE iff for all $b, b^{\prime} \in B, b \cap b^{\prime} \neq \emptyset$. It is well known that this is a necessary condition for Lockean Compatibility, Completeness, or Almost Completeness for $t>.5 .^{11}$ However it is not a sufficient condition for any of these properties as the following result shows:

Observation 1 For any $t>0$, there is a set of beliefs $B$ that is pairwise compatible but not Lockean Compatible at $t$.

Proof Let $W$ be the set

\footnotetext{
9 This is equivalent to saying $\langle W, P, c\rangle$ is a probability space. Note that, unlike in standard Dutch Book arguments, we are here stipulating that credences satisfy the axioms of probability theory.

${ }^{10}$ For two $n$-dimensional vectors, $\mathbf{x}=\left\langle x_{1} \ldots x_{n}\right\rangle$ and $\mathbf{y}=\left\langle y_{1} \ldots y_{n}\right\rangle$, their inner product, $\mathbf{x} \cdot \mathbf{y}$, is $x_{1} y_{1}+\cdots+x_{n} y_{n}$.

11 This is because if two propositions are disjoint then both their probabilities cannot be greater than .5 .
} 


\section{$\{\{i, j\}: i, j$ are positive integers less than or equal to $n$ s.t. $i \neq j\}$.}

Consider the set $B$ enumerated $b_{1} \ldots b_{n}$ such that $b_{k}=\{w \in W: k \in w\}$. Note that for any $i \neq j,\{i, j\}$ is a member of both $b_{i}$ and $b_{j}$ and no other element of $B$. So, $B$ is pairwise compatible and each element of $W$ is a member of exactly two elements of $B$. It follows that for any credence function $c$ :

$$
\sum_{i=1}^{n} c\left(b_{i}\right)=2 \sum_{w \in W} c(\{w\})=2 .
$$

It follows that for some $j$ between 1 and $n$ that $c\left(b_{j}\right) \leq \frac{2}{n}$. So if we choose $n$ large enough we can find a member of $B$ with an arbitrarily low credence. ${ }^{12}$

Note that since Lockean Compatibility is the weakest of our three notions, we also cannot use pairwise compatibility to give sufficient conditions for being Lockean Complete or Almost Lockean Complete. ${ }^{13}$

\section{Betting on Beliefs}

Dutch Book arguments traditionally assume that an agent's rational betting behavior is determined by their credences. Dutch Book arguments are used to argue for the thesis that a rational agent's credences are probabilistic (something we assumed above in the definition of credences). The leading assumption behind the Dutch Book argument is that credences determine a rational agent's betting preferences. The arguments typically assume some divisible good in which an agent's utility is linear (conventionally assumed to be dollars). Expected utility theory provides a standard way of linking rational betting dispositions to credences: a rational agent acts in order to maximize her utility relative to here credences. Thus, if offered a bet, an agent ought to be willing to take it only if she expects to gain by it or at least not lose by it. Indeed the very idea of credences or subjective probability is often considered to be determined by or to determine betting behavior. ${ }^{14}$ On this framework it is typically assumed that if an agent has a credence $y$ in a proposition $p$ then she ought to be willing to buy or sell a bet that pays out $\$ 1$ if $p$ is true and $\$ 0$ otherwise (we'll call this bet $a$ bet for $p$ ) for the price $\$ y$. This is because the agent's expected return from the bet is $\$ y$. (In the decision theoretic framework, the agent is also rationally obliged to buy the bet for any lower value and sell it for any higher value.) A Dutch Book is a way of exploiting these dispositions, by presenting an agent with a collection of bets she is willing to take which will result in a sure loss for the agent, and,

\footnotetext{
12 Thanks to Kenny Easwaran for noting and fixing a mistake here.

${ }^{13}$ Note that the combination of single premise closure (for every $b \in B$ if $p \supseteq b$ then $p \in B$ ) and pairwise compatibility also does not yield necessary and sufficient conditions for a Lockean complete sets of beliefs.

${ }^{14}$ As in Ramsey (1926).
} 
hence, a sure gain for the bookie (i.e. the person offering the bets). Traditional Dutch Book arguments show that, roughly, an agent will not be subject to a Dutch Book if and and only if her credences are probabilistically coherent. ${ }^{15}$

It is less straightforward to link all-or-nothing beliefs to betting behavior. The Lockean thesis, however, by providing a link between belief and credence suggests some ways of linking beliefs to betting behavior. On the Lockean thesis an agent ought to believe $p$ if and only if she assigns $p$ a credence greater than or equal to $t$. The agent, then, for each belief $b$ ought to be willing to buy a bet for $b$ for $\$ t$ (or a lower price), since her expected return of the bet is greater than or equal to zero. So we start with the assumption that there are some bets that a Lockean agent ought to be willing to buy (in any quantities). If there is a collection of such bets for an agent that guarantee her a loss at each world then the agent's beliefs $B$ is subject to a ONEWAY DuTCH Book with respect to $t$.

To state the notion of a one-way Dutch Book formally we will again use vector notation. For an agent's beliefs $b_{1}, \ldots, b_{o}$ we can use a non-negative $o$-dimensional vector $\mathbf{x}$, the stake vector, to represent the number of bets the agent buys for each proposition $b_{1}, \ldots, b_{o}$ (i.e. the agent buys $x_{i}$ bets for $b_{i}$ ). ${ }^{16} \mathrm{We}$ assume here that all bets are priced at $\$ t$ since that is the highest price that the Lockean view guarantees the agent will be willing to pay. At a world $w$ these bets lead to the payoff,

$$
\sum_{1 \leq i \leq o, w \in b_{i}} x_{i}(1-t)+\sum_{1 \leq i \leq o, w \notin b_{i}}-x_{i} t .
$$

By associating each world $w$ with an $o$-dimensional vector $\mathbf{w}$, such that

$$
w_{i}= \begin{cases}(1-t) & \text { if } w \in b_{i} \\ -t & \text { otherwise }\end{cases}
$$

the payoff at $w$ can be concisely stated as $\mathbf{w} \cdot \mathbf{x}$. A collection of beliefs $b_{1}, \ldots, b_{o}$ is subject to a one-way Dutch Book just in case there is some vector of non-negative stakes $\mathbf{x}$ such that for all worlds $w, \mathbf{x} \cdot \mathbf{w}<0$.

A one-way Dutch Book only takes advantage of the beliefs $B=b_{1}, \ldots, b_{o}$ the agent has, it does not exploit those propositions she does not belief, i.e. $P \backslash B$. On the Lockean view, the agent must have less than $t$ credence in the propositions not in $B$. She, thus, ought to be willing to sell $\$ 1$ bets on each proposition in $P \backslash B$ for $\$ t$ each. A two-way Dutch Book is a collection of bets in $B$ that the agent is willing to buy at $\$ t$ and a collection of bets in $P \backslash B$ she is willing to sell at $\$ t$ that together guarantee her a loss.

We will formally state the notion of two-way Dutch Book in vector notation. Let a non-negative $n$-dimensional vector $\mathbf{x}$ represent the stakes an agent takes in bets costing $\$ t$ on the propositions $p_{1}, \ldots, p_{n}$ as follows: if $p_{i}$ is one of the agent's beliefs then

15 See Pettigrew (2019) for a recent review of these arguments.

16 We are using an $o$-dimensional vector to represent a function from $B$ to non-negative stakes. 
she buys $x_{i}$ bets, if $p_{i}$ is not one of the agent's beliefs then she sells $x_{i}$ bets. ${ }^{17}$ That $\mathbf{x}$ is required to be nonnegative captures the fact that the choice of buying or selling a bet in a proposition is determined by whether or not the proposition is believed by the agent.

The payout an agent gets for stakes $\mathbf{x}$ with the set of beliefs $B$ at a world $w$ is:

$$
\sum_{p_{i} \in B, p_{i} \in w} x_{i}(1-t)+\sum_{p_{i} \in B, p_{i} \notin w}-x_{i} t+\sum_{p_{i} \notin B, p_{i} \in w}-x_{i}(1-t)+\sum_{p_{i} \notin B, p_{i} \notin w} x_{i} t .
$$

We can represent this as $\mathbf{x} \cdot \mathbf{w}$, where the $n$-dimensional vector $\mathbf{w}$ is defined as follows:

$$
w_{i}=\left\{\begin{array}{ll}
1-t & \text { if } p_{i} \in B, p_{i} \in w \\
-t & \text { if } p_{i} \in B, p_{i} \notin w \\
-(1-t) & \text { if } p_{i} \notin B, p_{i} \in w \\
t & \text { if } p_{i} \notin B, p_{i} \notin w
\end{array} .\right.
$$

An agent is subject to a two-way Dutch Book if there is a non-negative $n$-dimensional stake vector $\mathbf{x}$ such that for each world $w, \mathbf{x} \cdot \mathbf{w}<0$. In other words, there is a set of bets the agent's beliefs rationalize that leads to a loss at each world.

\section{Scoring Beliefs for Accuracy}

Another perspective associates sets of beliefs with scores, depending on the accuracy of the beliefs in the set. The picture here is that an agents' beliefs $B$ are scored for their accuracy. Scoring all-or-nothing beliefs is simpler conceptually than scoring graded beliefs: while saying whether a real-valued confidence in a proposition is right or wrong at a world is a tricky business, saying whether a belief is right or wrong is straightforward. ${ }^{18}$ For this reason, we'll jump right into how to score sets of all-or-nothing beliefs without comparison to the scoring of graded beliefs.

We'll consider a scoring system for beliefs following Easwaran and Fitelson (2015), Easwaran (2016) and Dorst (2019). The crucial assumption made in this literature is that we assign scores to an agent for their total beliefs at a world by assigning numerical scores to individual propositions believed and summing those scores. ${ }^{19}$ We will further assume that at a world $w$ if a belief in a proposition $p_{i}$ turns out to be correct (i.e. $w \in p_{i}$ ) then the agent gets a certain non-negative score $r_{i} \geq 0$, and if it is incorrect (i.e. $w \notin p_{i}$ ) the agent gets a non-positive score, $s_{i} \leq 0$. Note that if an agent fails to believe a proposition there is no score associated with that proposition. We will further assume that the ratio between the reward for true belief

\footnotetext{
17 Note that we reuse $\mathbf{x}$ and $\mathbf{w}$ for both $o$-dimensional and $n$-dimensional vectors. This abuse of notation is justified because of the similar construction and role these vectors play across different arguments and definitions. It will always be clear, I hope, which one we are discussing.

18 See Gneiting and Raftery (2007) for a review of scoring rules for graded beliefs.

19 The additivity assumption, that the total score for one's beliefs is just the sum of the individual scores, is defended by Dorst (2019).
} 
and penalty for false belief is constant across propositions so that for any two propositions $p_{i}$ and $p_{j}, \frac{r_{i}}{s_{i}}=\frac{r_{j}}{s_{j}}$, if all of $r_{i}>0, s_{i}>0, r_{j}>0$ and $s_{j}$ are non-zero. ${ }^{20}$ We also assume that for any $p_{i}, r_{i}=0$ iff $s_{i}=0$. On this system of scoring beliefs, at a world $w$ a set of beliefs $B$ gets the score

$$
S(w, B)=\sum_{w \in p_{i}, p_{i} \in B} r_{i}+\sum_{w \notin p_{i}, p_{i} \in B} s_{i} .
$$

We now rephrase this scoring system in a way that makes more transparent its connection to Lockeanism. Using the threshold $t$, for reasons that will become apparent, let $t=\frac{-s_{i}}{r_{i}-s_{i}}$, for some $i$ such that $r_{i}>0$. Note that $t$ does not depend on the choice of $i$ and $0 \leq t \leq 1$. Let $x_{i}=r_{i}-s_{i}$. Note that $-t x_{i}=s_{i}$ and $(1-t) x_{i}=r_{i}$. We will refer to $x_{i}$ as the WEIGHT on the score for proposition $p_{i}$, as the higher an $x_{i}$ is the more extreme a score the proposition $p_{i}$ has, relative to a fixed $t$. Note that $x_{i}$ is always non-negative. Let $\mathbf{x}$ be the $m$-dimensional vector whose $i$ th entry is $x_{i}$. Given this mapping, it is clear that any scoring system, as defined in the previous paragraph, can be fully specified by giving a $t: 0<t \leq 1$ and a non-negative $n$-dimensional vector $\mathbf{x}$. In what follows, we will specify scoring systems in terms of $t$ and $\mathbf{x}$ without loss of generality.

Let $\mathbf{w}$, again, be the $o$-dimensional vector defined in (1) on p. 8. Let $\mathbf{x}$, the weight vector, be a non-negative $o$-dimensional vector giving the weights on a scoring system for the propositions $b_{1}, \ldots, b_{n}{ }^{21}$ The agent's total score at $w, S(w, B)$ can be concisely stated in vector notation as $\mathbf{w} \cdot \mathbf{x}$.

Since we have a scoring system for beliefs, we can apply some standard notions from decision theory to the choice of belief sets. A choice of a set of beliefs $B$ is a SURE LOSS if for every world $w, S(w, B)<0$. A belief set $B$ is RATIONAL for an agent given a credence function $c$ if and only if there is no other set $B^{\prime}$ such the expected score of $B$ on $c, E\left(S\left(w, B^{\prime}\right)\right)$, is greater than the expected score of $B^{\prime}$ on $c, E\left(S\left(w, B^{\prime}\right)\right)$ .${ }^{22}$ The choice of one set of beliefs $B$ STRICTLY DOMINATES another choice of beliefs, $B^{\prime}$ if for all worlds $w, S(w, B)>S\left(w, B^{\prime}\right)$. A set of beliefs $B$ WEAKLY DOMINATES another set of beliefs, $B^{\prime}$, if for all worlds $w, S(w, B) \geq S\left(w, B^{\prime}\right)$ and there is some world $w^{\prime}$ such that $S(w, B)>S\left(w, B^{\prime}\right)$. Note that all of these notions are only defined relative to the scoring system, and hence the weight vector $\mathbf{x}$ (for all propositions) as well as the threshold $t$.

\footnotetext{
${ }^{20}$ We can remove this assumption, but then we need to relate this scoring system to a slightly different definition of Lockeanism in which there is a different threshold for belief in each proposition, see Easwaran (2016, Appendix F), Dorst (2019), for details.

21 These are the only weights necessary for determining the score for the set of beliefs $B$, which is why we do not use our original $n$-dimensional weight vector also called $\mathbf{x}$. Again the reuse of the $\mathbf{x}$ and $\mathbf{w}$ for both $o$-dimensional and $n$-dimensional vectors is to emphasize their parallel roles.

22 Where $E(S(w, B))=\sum_{w \in W} c(\{w\}) S(w, B)$.
} 


\section{Connecting the Three Perspectives}

First, we will draw some connections between properties of Dutch Books and properties of scoring systems on our framework.

Observation 2 For any set of worlds $W=w_{1}, \ldots, w_{m}$, set of beliefs $B=b_{1}, \ldots, b_{o}$, and positive real number $t \leq 1$, the following two statements are equivalent.

(a) The agent holding $B$ is subject to a one-way Dutch Book at threshold $t$.

(b) At some weight vector $\mathbf{x}$ the agent holding $B$ will realize a sure loss (i.e. a loss at all worlds) at threshold $t$.

Proof Corresponding to each world $w$ there is an $o$-dimensional vector $\mathbf{w}$ as defined in (1) on p. 8. Note that $\mathbf{x} \cdot \mathbf{w}$ is both the payoff at $w$ frzzaom holding bets on $B$ with stake vector $\mathbf{x}$ with threshold $t$ and the score one gets at $w$ for having beliefs $B$ with weight vector $\mathbf{x}$ and threshold $t$. So, both (a) and (b) are equivalent to the statement that there is some non-negative $o$-dimensional vector $\mathbf{x}$ such that for every world $w$, $\mathbf{x} \cdot \mathbf{w}<0$.

Another, less obvious, connection between scoring and betting can be made as follows:

Observation 3 For any set of worlds $W=w_{1}, \ldots, w_{m}$, set of beliefs $B=b_{1}, \ldots, b_{o}$, and positive real number $t \leq 1$ the following two statements are equivalent.

(a) The agent holding $B$ is subject to a two-way Dutch Book at threshold $t$.

(b) The set of beliefs $B$ is strictly dominated by another set of beliefs $B^{\prime}$ on the scoring system with threshold $t$ and some weight vector $\mathbf{x}$.

Proof We will start by showing (a) implies (b): Let $\mathbf{w}$ be a non-negative $n$-dimensional vector as defined in (2) on p. 9. Since $B$ is subject to a two-way Dutch Book we know that there is some weight vector $\mathbf{x}$ such that for every world $w, \mathbf{w} \cdot \mathbf{x}<0$. In scoring terms $\mathbf{w} \cdot \mathbf{x}$ represents the score at world $w$ for holding $B$ minus the score for holding $P \backslash B$. So $P \backslash B$ strictly dominates $B$ on weights $\mathbf{x}$.

Now we will show that (b) implies (a). If $B$ is strictly dominated we know that there is some other set of bets $B^{\prime}$ and some set of non-negative weights $\mathbf{x}$ such that for every world $w$ the score for holding $B$ with $t$ and $\mathbf{x}$ is strictly less then the score for holding $B^{\prime}$. Now define $\mathbf{x}^{\prime}$ such that,

$$
x_{i}^{\prime}= \begin{cases}x_{i} & \text { if } b_{i} \in B \backslash B^{\prime} \cup B^{\prime} \backslash B \\ 0 & \text { otherwise }\end{cases}
$$

It is straightforward to see that on $\mathbf{x}^{\prime}, B^{\prime}$ strictly dominates $B$ : since $B$ strictly dominates $B^{\prime}$ on $\mathbf{x}$, and $\mathbf{x}^{\prime}$ is like $\mathbf{x}$ except for not weighting propositions held in common by $\mathbf{B}$ and $\mathbf{B}^{\prime}$. We can also see that $P \backslash B$ strictly dominates $B$ on $\mathbf{x}^{\prime}$ since the scores for $P \backslash B$ and $B^{\prime}$ on $\mathbf{x}^{\prime}$ are equal at every world. Since $\mathbf{x}^{\prime} \cdot \mathbf{w}$ is the score for holding $B$ 
minus the score for holding $P \backslash B$, it must be negative for every $w$. Therefore $\mathbf{x}^{\prime}$ are the stakes for a two-way Dutch Book.

We have just seen the close relationship between the betting dominance and accuracy dominance of a set of beliefs $B$. Now we can make connections between these and Lockeanism.

We begin by reviewing a connection between the scoring system and Lockeanism that has been established in the literature. The central result in the literature is the following. ${ }^{23}$

Observation 4 Given a scoring system with threshold $t$, a set of beliefs is rational relative to some credences $c$ if it is Almost Lockean Complete with respect to $t$.

Proof We give a sketch, referring the reader to Dorst (2019) and Easwaran (2016) for more detail. Given the additivity of our scoring system and of expectations, an agent maximizes expected score for the total set of beliefs, by making the 'best' choice for each proposition $p$ whether to belief it or not. If the scoring system assigns a positive weight to $p$ this requires believing $p$ only if $c(p) \geq t$ and whenever $c(p)>t$, hence being Almost Lockean Complete. Note that since weights are not required to be positive the converse fails.

Relating rationality, which is expressed in terms of credences, to Lockeanism is straightforward as the previous result showed. The notion of a sure loss and the dominance relations are not stated in terms of credences and so their relationship to Lockeanism less transparent. We begin with a negative observation, due to Easwaran (2016). Suppose a belief set $B$ is not weakly dominated on the scoring system with weights $\mathbf{x}$ and threshold $t$. This does not entail that $B$ is (Almost) Lockean Complete. A simple example suffices, where there are two worlds $w_{1}, w_{2}$ and threshold .6 and all propositions have weight 1 except $\left\{w_{1}\right\}$ which has weight 100 . In this case the belief set $\left\{\left\{w_{1}\right\},\left\{w_{2}\right\},\left\{w_{1}, w_{2},\right\}\right\}$ is not weakly dominated. It yields score 40 at $w_{1}$ and -59.2 at $w_{2}$. Dropping $\left\{w_{1}\right\}$ would reduce the score at $w_{1}$ while dropping $\left\{w_{2}\right\}$ would reduce the score at $w_{2}$, while dropping both would reduce the score at $w_{1}$. However, given the threshold of .6, an Almost Lockean Complete set of beliefs cannot include two pairwise incompatible propositions such as a $\left\{w_{1}\right\}$ and $\left\{w_{2}\right\}$.

We now turn to our main results: characterizations of Lockean Compatibility and Almost Lockean Completeness in betting and scoring terms.

Here is our first result:

Theorem 1 The following three statements are equivalent.

(a) The agent holding $B$ is not subject to a one-way Dutch Book at threshold $t$.

${ }^{23}$ See Easwaran (2016) and Dorst (2019). Both generalize to cases where $t$ is proposition dependent. 
(b) There is no weight vector $\mathbf{x}$ such that the agent holding $B$ will realize a sure loss at threshold $t$.

(c) B is Lockean Compatible with threshold $t$.

Proof As we have already showed that (a) and (b) are equivalent in Observation 2, we will only need to show that (c) is equivalent to those two. To do so we will need to represent the entire situation in terms of matrix-vector multiplication.

Consider the $m \times o$ matrix $\mathbf{A}$, constructed so that each row $i$ consists of the row vector $\mathbf{w}_{i}$ corresponding to the world $w_{i}$ as defined in (1) on p. 8.

$$
\mathbf{A}=\left[\begin{array}{c}
\mathbf{w}_{1} \\
\vdots \\
\mathbf{w}_{m}
\end{array}\right]
$$

Or equivalently we can directly define $\mathbf{A}$ as follows:

$$
\mathbf{A}=\left[\begin{array}{ccc}
a_{11} & \ldots & a_{1 o} \\
\ldots & \ldots & \ldots \\
a_{m 1} & \ldots & a_{m n}
\end{array}\right],
$$

where

$$
a_{i j}=\left\{\begin{array}{cl}
1-t & \text { if } w_{i} \text { is in } b_{j} \\
-t & \text { otherwise }
\end{array}\right.
$$

Note that if we multiply $\mathbf{A}$ by an $o$-dimensional column vector $\mathbf{x}$ we get the following:

$$
A \mathbf{x}=\left[\begin{array}{c}
\mathbf{w}_{1} \cdot \mathbf{x} \\
\vdots \\
\mathbf{w}_{\mathbf{m}} \cdot \mathbf{x}
\end{array}\right]
$$

We can see, then, that iff there is no non-negative $\mathbf{x}$ such that $\mathbf{A x}<0$ then (a) and (b) hold. ${ }^{24}$ So, to put it more compactly, (a) and (b) are equivalent to the following holding:

$$
\nexists \mathbf{x} \geq 0: \mathbf{A x}<0
$$

Consider an $m$-dimensional vector corresponding to some credence function $\mathbf{c}$, as in Sect. 3. Now consider multiplying such a vector in row format by $\mathbf{A}$ as follows:

${ }^{24}$ Note that, as is standard, $\mathbf{v}<0$ means each coordinate of $\mathbf{v}, v_{1} \ldots v_{k}$, is less than zero. 


$$
\begin{aligned}
\mathbf{c}^{T} \mathbf{A} & =\left[c_{1}, \ldots, c_{m}\right]\left[\begin{array}{ccc}
a_{11} & \ldots & a_{1 o} \\
\ldots & \ldots & \ldots \\
a_{m 1} & \ldots & \ldots
\end{array}\right] \\
& =\left[\sum_{i: w_{i} \in b_{1}} c_{i}(1-t)-\sum_{i: w_{i} \notin b_{1}} c_{i} t, \ldots, \sum_{i: w_{i} \in b_{o}} c_{i}(1-t)-\sum_{i: w_{i} \notin b_{o}} c_{i}(t)\right] \\
& =\left[c\left(b_{1}\right)(1-t)-\left(1-c\left(b_{1}\right)\right)(t), \ldots, c\left(b_{o}\right)(1-t)-\left(1-c\left(b_{o}\right)\right)(t)\right]
\end{aligned}
$$

We can see that the $i$ th coordinate of $\mathbf{c}^{T} \mathbf{A}$ is greater than or equal to 0 iff $c\left(b_{i}\right) \geq t$. Another way of seeing this is to note that the $i$ th column of $\mathbf{A}$ is the vector $\mathbf{b}_{i}$ minus the vector $\mathbf{t}$ which has value $t$ everywhere. ${ }^{25}$ Thus, the $i$ th entry of $\mathbf{c}^{T} \mathbf{A}$ is $\mathbf{c} \cdot(\mathbf{p}-\mathbf{t})$ $=\mathbf{c} \cdot \mathbf{p}-t$. If this is greater than or equal to 0 , then $c(p) \geq t$.

We can now compactly state what it is for a set of beliefs to satisfy (c) as follows:

$$
\exists \mathbf{y} \geq \mathbf{0}: \mathbf{y} \neq \mathbf{0} \text {, and } \mathbf{y A} \geq 0
$$

Note that we do not require $\mathbf{y}$ to sum to 1 in (4). However, it is easy to see that (4) is equivalent to

$$
\exists \mathbf{y} \geq \mathbf{0}, \mathbf{y} \neq \mathbf{0}, \mathbf{y} \mathbf{A} \geq 0, \text { and } \sum_{i=1}^{m} y_{i}=1,
$$

since multiplying $\mathbf{y}$ by a positive scalar does not affect any of the inequalities.

So all we need to show is that (3) and (4) are equivalent statements about the matrix $\mathbf{A}$, which is the content of the following theorem from linear algebra, whose proof, using Farkas's Lemma, is in the appendix.

Theorem 2 Let $\mathbf{A}$ be any $m \times n$ matrix, then:

$$
(\nexists \mathbf{x} \geq \mathbf{0}: \mathbf{A x}<\mathbf{0}) \leftrightarrow(\exists \mathbf{y} \geq \mathbf{0}: \mathbf{y} \neq 0 \text { and } \mathbf{y A} \geq \mathbf{0})
$$

The next theorem relates the notion of strict dominance to the scoring and betting frameworks.

Theorem 3 The following three statements are equivalent:

(a) There is no two-way Dutch Book on B.

(b) There is no weight vector $\mathbf{x}$ on which B is strictly dominated.

(c) B is Almost Lockean Complete.

Proof We have already established that $(a)$ and $(b)$ are equivalent in Observation 3. Consider the following $m \times n$ matrix $\mathbf{D}$.

\footnotetext{
25 Vectors for propositions were defined in Sect. 3 and simply are $m$-dimensional vectors that are 1 at index $i$ when proposition is true at $w_{i}$ and 0 otherwise.
} 


$$
\mathbf{D}=\left[\begin{array}{ccc}
d_{11} & \ldots & d_{1 n} \\
\ldots & \ldots & \ldots \\
d_{m 1} & \ldots & d_{m n}
\end{array}\right]
$$

where,

$$
d_{i j}=\left\{\begin{array}{ll}
1-t & \text { if } w_{i} \in p_{j} \text { and } p_{j} \in B \\
-(1-t) & \text { if } w_{i} \in p_{j} \text { and } p_{j} \notin B \\
-t & \text { if } w_{i} \notin p_{j} \text { and } p_{j} \in B \\
t & \text { if } w_{i} \notin p_{j} \text { and } p_{j} \notin B
\end{array} .\right.
$$

Note that the $i$ th row of $\mathbf{D}$ corresponds to the vector $\mathbf{w}_{i}$ associated with the world $w_{i}$ as defined in (2) on p. 9. Given an $n$-dimensional non-negative weighting vector $\mathbf{x}, \mathbf{x} \cdot \mathbf{w}_{i}<0$ iff taking stakes $\mathbf{x}$ on the bets associated with the belief set $B$ leads to a loss at $w_{i}$. Since

$$
\mathbf{D x}=\left[\begin{array}{c}
\mathbf{w}_{1} \cdot \mathbf{x} \\
\vdots \\
\mathbf{w}_{\mathbf{n}} \cdot \mathbf{x}
\end{array}\right]
$$

the statement,

$$
\nexists \mathbf{x}: \mathbf{x} \geq 0 \text { and } \mathbf{D x}<0
$$

is equivalent to saying that there is no two-way Dutch Book on $B$ (at the threshold $t$ ), i.e. that $(a)$ and $(b)$ are true.

Considered column-wise, the $j$ th column of $\mathbf{D}$ is $\mathbf{p}_{\mathbf{j}}-\mathbf{t}$ if $p_{j} \in B$ and $-\left(\mathbf{p}_{\mathbf{j}}-\mathbf{t}\right)$ if $p_{j} \notin B$, where $\mathbf{p}_{\mathbf{j}}$ is the $m$-dimensional proposition vector as defined in Sect. 3 and $\mathbf{t}$ is the $m$-dimensional vector with value $t$ at all coordinates. There being a credence function that is Almost Lockean Complete, (c) above, is thus equivalent to there being a credence vector $\mathbf{c}$ such that $\mathbf{c}^{T} D \geq 0$. This is equivalent to this simplified condition:

$$
\exists \mathbf{y}: \mathbf{y} \geq \mathbf{0}, \mathbf{y} \neq \mathbf{0} \text {, and } \mathbf{y D} \geq 0 \text {. }
$$

To complete our proof we note that Theorem 2 establishes that (5) and (6) are equivalent statements about D. It follows that (a), (b), and (c) are equivalent.

The following conjecture from Easwaran (2016) follows directly from Theorem 3.

Lemma 1 (Easwaran's conjecture) If for all positive weight vectors $\mathbf{x}, B$ is not weakly dominated by some $B^{\prime}$, then $B$ is Almost Lockean Complete.

Proof Given Theorem 3 what we can show is that if $B$ is not weakly dominated on any positive weighting function, then there is no non-negative weighting function on which $B$ is strictly dominated. We will prove the contrapositive. Suppose $B$ is strictly 
dominated by $B^{\prime}$ on some non-negative weight vector $\mathbf{x}$. Let $\mathbf{x}^{\prime}$ be a strictly positive weight vector such that

$$
x_{i}^{\prime}=\left\{\begin{array}{ll}
x_{i} & \text { if } x_{i}>0 \\
1 & \text { otherwise }
\end{array} .\right.
$$

Let $B^{\prime \prime}$ be the following belief set:

$$
B^{\prime \prime}=\left\{p_{i}: p_{i} \in B^{\prime} \text { and } x_{i} \neq 0\right\} \cup\left\{p_{i}: p_{i} \in B \text { and } x_{i}=0\right\} .
$$

We can see that since $B^{\prime}$ strictly dominates $B$ on $\mathbf{x}, B^{\prime \prime}$ will strictly dominate $B$ on $\mathbf{x}^{\prime}$. So $B$ is weakly dominated on the positive weight vector $\mathbf{x}^{\prime}$.

Note that the converse of Lemma 1 fails. $B$ can be Almost Lockean Complete but still weakly dominated on some strictly positive weighting function. This is because if the credence function $c$ assigns 0 to the proposition $\left\{w_{1}\right\}$ and $t$ to $\left\{w_{2}\right\}$ then $B$ might include $\left\{w_{2}\right\}$ and not include $\left\{w_{1}, w_{2}\right\}$ and still be Almost Lockean Complete. It will be weakly dominated (on a positive weighting vector) by a variation $B^{\prime}$ that is like $B$ except it includes $\left\{w_{1}, w_{2}\right\}$ but not $\left\{w_{2}\right\}$.

\section{Discussion}

Theorems 1 and 3 provide characterizations of Lockean belief sets (both Lockean Compatible and Almost Lockean Complete) in terms of the betting and accuracy frameworks.

As motivations for the Lockean thesis the significance of the results depends (a) on how natural the scoring and betting frameworks used are and (b) how compelling the conditions put on collections of beliefs relative to these frameworks are. With respect to the betting framework: the natural question to ask is whether we are rationally obliged to bet on our beliefs, or against our non-beliefs, in the ways specified in Sect. 4. While it is natural to think that one is rationally obliged to bet on one's full beliefs, it is perhaps less obvious that one has to bet against one's nonbeliefs. Certainly though, if such betting dispositions are normatively linked to beliefs, the requirement that we not be subject to Dutch Books is a plausible condition to put on rational beliefs. ${ }^{26}$ With respect to the scoring framework: if the idea of scoring beliefs for accuracy is attractive in general, then the framework used is quite natural. ${ }^{27}$ However, we might ask why rational agents ought to choose belief sets that are not either (a) subject to a sure loss on some weighting vector, or (b) strictly dominated on some weighting vector. It is clear that if we know what the actual weighting vector is we should avoid a sure loss/strict domination on that vector. But why should we avoid it on all weighting vectors? Two thoughts are possible here:

\footnotetext{
${ }^{26}$ See Pettigrew (2019) for a recent review and discussions of some of the philosophical questions about whether rational agents are obliged to make bets on their graded beliefs.

27 See in particular Easwaran (2016) and Dorst (2019) for robust presentation and defences of it.
} 
one is that we have uncertainty over what the right weights are and we are treating that uncertainty conservatively by ensuring we are okay (i.e. our choice is not dominated) no matter what the weighting vector is, the other is that there is unquantifiable uncertainty about what the weights are (since perhaps we don't have graded beliefs at all) and avoiding sure loss or weakly dominated beliefs on any weights is the best policy. ${ }^{28}$

\section{Further Directions}

\subsection{Connections to Wald's Complete Class Theorem}

I would conjecture that an alternative characterization of Almost Lockean Completeness can be found using Wald's Complete Class Theorem. ${ }^{29}$ Wald's Complete Class Theorem establishes that in a certain class of decision problems any strategy that is not weakly dominated is a rational strategy (i.e. one maximizing expected reward subject to her credences). Wald's Complete Class theorem does not directly apply to the choice problem here: for, as I noted in Sect. 6, there are some belief sets that are not weakly dominated at some weighting function but that are also not Almost Lockean Complete. The reason Wald's Complete Class theorem does not apply is that it requires the class of strategies to be convex: i.e. if two strategies are available to an agent then any probabilistic mix of them also is. If we had allowed mixed strategies in our decision problem, then the Complete Class Theorem would apply and any strategy that was not weakly dominated by any mixed strategy would be Almost Lockean Complete. However, the main point of modeling outright belief is to have a notion of belief that contrasts with graded belief. If we allowed mixed strategies involving outright belief, it is not so clear that we have not introduced an analogue of graded beliefs. Nonetheless, I conjecture that by applying Wald's Complete Class Theorem we can show that outright beliefs that are Almost Lockean Complete will be just those that are not weakly dominated by any mixture of other outright beliefs. Future work might also investigate how we ought to think about mixed strategies in a system of outright belief and how such mixed strategies relate to graded beliefs.

\subsection{Extensions to Other Scoring Systems}

Note that we assumed here that at the threshold $t$, the betting odds, and the scoring system ratios of cost to rewards, were the same for every proposition. A simple extension of these results could handle cases in which the odds are allowed to vary for each proposition. ${ }^{30}$ In addition to this last extension, future work might also

\footnotetext{
${ }^{28}$ I am grateful to Kevin Dorst for this last point.

29 I am grateful to Gary Chamberlain here for pointing out the relevance of Wald's Complete Class Theorem. See, e.g., Ferguson (1967) for details.

30 See Easwaran (2016) and Dorst (2019) for the formal description of such a scoring system. The basic idea is that a $n$-dimensional vector $\mathbf{t}$ would specify the threshold for each proposition. The results here, I believe, could easily be extended to these systems.
} 
explore other scoring systems and see whether they also give rise to Lockean patterns of beliefs. Most interesting, I think, would be to see what can be found when the additivity assumption is weakened.

\subsection{Connection to Another Characterization}

In this paper I characterized the Lockean belief sets by way of their relationship to bets and accuracy scoring systems. Fernando (1998, Theorem 4, p. 230) gives a different characterization of Lockeanism that is based on qualitative properties of sets of beliefs. ${ }^{31}$ It would be interesting to explore the connections between this result and those presented here.

\section{Appendix: Proof of Theorem 2}

Theorem 2 Let $\mathbf{A}$ be any $m \times n$ matrix, then:

$$
(\nexists \mathbf{x} \geq \mathbf{0}: \mathbf{A x}<\mathbf{0}) \leftrightarrow(\exists \mathbf{y} \geq \mathbf{0}: \mathbf{y} \neq 0 \text { and } \mathbf{y} \mathbf{A} \geq \mathbf{0}) .
$$

Proof We start with this widely used variant of Farkas's Lemma: ${ }^{32}$

$$
(\nexists \mathbf{x} \geq \mathbf{0}: \mathbf{A x} \geq \mathbf{b}) \leftrightarrow(\exists \mathbf{y} \leq 0: \mathbf{y A} \geq \mathbf{0} \text { and } \mathbf{y b}<0) .
$$

We can get the following by taking a universal instantiation of the both sides of the biconditional. (i.e. going from ' $\mathrm{p}$ iff q' $\rightarrow$ '(for all x p) iff (for all x q)').

$$
(\forall \mathbf{b}>0, \nexists \mathbf{x} \geq \mathbf{0}: \mathbf{A x} \geq \mathbf{b}) \leftrightarrow(\forall \mathbf{b}>\mathbf{0}, \exists \mathbf{y} \leq 0: \mathbf{y A} \geq 0 \text { and } \mathbf{y b}<0)
$$

This can be simplified to:

$$
(\nexists \mathbf{x} \geq \mathbf{0}: \mathbf{A x}>\mathbf{0}) \leftrightarrow(\exists \mathbf{y} \leq \mathbf{0}: \mathbf{y} \neq \mathbf{0} \text { and } \mathbf{y A} \geq \mathbf{0}) .
$$

We can then switch signs on the right-hand side to get:

$$
(\nexists \mathbf{x} \geq \mathbf{0}: \mathbf{A x}>\mathbf{0}) \leftrightarrow(\exists \mathbf{y} \geq \mathbf{0}: \mathbf{y} \neq 0 \text { and } \mathbf{y A} \leq \mathbf{0}) .
$$

Theorem 2 follows immediately (by substitution of $\mathbf{A}$ with $-\mathbf{A}$ ):

$$
(\nexists \mathbf{x} \geq \mathbf{0}: \mathbf{A x}<\mathbf{0}) \leftrightarrow(\exists \mathbf{y} \geq \mathbf{0}: \mathbf{y} \neq 0 \text { and } \mathbf{y A} \geq \mathbf{0})
$$

\footnotetext{
31 Wes Holliday pointed out to me that Fernando's result can also be derived as a direct consequence of a result in Adams (1965, Theorem 1.4, p. 213).

32 For statements and proof see Strang (2006, pp. 441-442), Matoušek, J. \& Gärtner (2007, pp. 89-92), and the wikipedia entry on Farkas's lemma (Wikipedia contributors 2019).
} 
Acknowledgements I am grateful to audiences at London School of Economics and University of Amsterdam, Gary Chamberlain, Nilanjan Das, Kevin Dorst, Kenny Easwaran, Branden Fitelson, Wes Holliday, Matt Mandelkern, Richard Pettigrew and Linda Rothschild for comments and discussion. Special thanks to an anonymous referee at Review of Symbolic Logic who gave extremly helpful and extensive comments on three drafts of the paper.

Open Access This article is licensed under a Creative Commons Attribution 4.0 International License, which permits use, sharing, adaptation, distribution and reproduction in any medium or format, as long as you give appropriate credit to the original author(s) and the source, provide a link to the Creative Commons licence, and indicate if changes were made. The images or other third party material in this article are included in the article's Creative Commons licence, unless indicated otherwise in a credit line to the material. If material is not included in the article's Creative Commons licence and your intended use is not permitted by statutory regulation or exceeds the permitted use, you will need to obtain permission directly from the copyright holder. To view a copy of this licence, visit http://creativecommons.org/licen ses/by/4.0/.

\section{References}

Adams, E. W. (1965). Elements of a theory of inexact measurement. Philosophy of Science, 32(3/4), 205-228. https://doi.org/10.1086/288044.

Brier, G. W. (1950). Verification of forecasts expressed in terms of probability. Monthly Weather Review, 78(1), 1-3. https://doi.org/10.1175/1520-0493.

de Finetti, B. (1974). Theory of probability (Vol. 1). Wiley.

Dorst, K. (2019). Lockeans maximize expected accuracy. Mind, 128, 175-211.

Easwaran, K. (2016). Dr. Truthlove or: How I learned to stop worrying and love Bayesian probabilities. Noûs, 50(4), 816-853. https://doi.org/10.1111/nous.12099

Easwaran, K., \& Fitelson, B. (2015). Accuracy, coherence, and evidence. Oxford Studies in Epistemology, 5, 61-96.

Ferguson, T. S. (1967). Mathematical Statistics. A decision theoretic approach. Academic Press.

Fernando, T. (1998). In conjunction with qualitative probability. Annals of Pure and Applied Logic, 92(3), 217-234

Foley, R. (1992). The epistemology of belief and the epistemology of degrees of belief. American Philosophical Quarterly, 29(2), 111-124.

Gneiting, T., \& Raftery, A. E. (2007). Strictly proper scoring rules, prediction, and estimation. Journal of the American Statistical Association, 102(477), 359-378. https://doi.org/10.1198/0162145060 00001437.

Hawthorne, J., \& Bovens, L. (1999). The preface, the lottery, and the logic of belief. Mind, 108(430), 241-264.

Joyce, J. M. (1998). A nonpragmatic vindication of probabilism. Philosophy of Science, 65(4), 575-603.

Leitgeb, H. (2014). The stability theory of belief. Philosophical Review, 123(2), 131-171.

Matoušek, J. \& Gärtner, B. (2007). Understanding and Using Linear Programming. Springer.

Pettigrew, R. (2016a). Accuracy and the laws of credence. Oxford University Press.

Pettigrew, R. (2016b). Jamesian epistemology formalised: An explication of 'The Will to Believe'. Episteme, 13(3), 253-268.

Pettigrew, R. (2019). The Dutch Book Arguments. Cambridge University Press.

Ramsey, F. P. (1926). "Truth and Probability", in Foundations of Mathematics and other Essays, R. B. Braithwaite (ed.), London: Kegan, Paul, Trench, Trubner, \& Co., 1931, 156-198.

Strang, G. (2006). Linear algebra and its applications, 4th edn. Thomson Brooks/Cole.

Wikipedia contributors. (2019). 'Farkas' lemma-Wikipedia, the free encyclopedia. https://en.wikipedia. org/w/index.php?title=Farkas\%27_lemma\&oldid=879978648. [Online; accessed 6-February-2019].

Publisher's Note Springer Nature remains neutral with regard to jurisdictional claims in published maps and institutional affiliations. 